\title{
Asociación entre los niveles de estrés y depresión y la adhesión al tratamiento en personas seropositivas al VIH en Hermosillo, México
}

\author{
Julio Alfonso Piña López ${ }^{1}$, Mariana Dávila Tapia, ${ }^{1}$ Juan José \\ Sánchez-Sosa, ${ }^{2}$ Carlos Togawa ${ }^{3}$ y Óscar Cázares Robles ${ }^{3}$
}

Forma de citar Piña López JA, Dávila Tapia M, Sánchez-Sosa JJ, Togawa C, Cázares Robles O. Asociación entre los
niveles de estrés y depresión y la adhesión al tratamiento en personas seropositivas al VIH en Hermoniveles de estrés y depresión y la adhesión al tratamiento en

RESUMEN Objetivos. Evaluar la asociación entre las variables relacionadas con el estrés, los motivos y la depresión en personas seropositivas al VIH y la adhesión al tratamiento, y analizar su consistencia según un modelo psicológico teórico.

Métodos. Estudio transversal con la participación de 25 mujeres y 39 hombres seropositivos al VIH atendidos en el Centro Ambulatorio para la Prevención y Atención a VIH/Sida e Infecciones de Transmisión Sexual, en Hermosillo, Sonora, México. Se exploraron las variables psicológicas y el grado de adhesión al tratamiento, las situaciones vinculadas con el estrés y el grado de depresión. Se elaboraron índices de las variables de interés asociadas con el estrés, los motivos y la depresión. La asociación entre las variables se determinó mediante regresión múltiple.

Resultados. En el mes previo al estudio, 65,6\% de los 64 participantes informó haber seguido fielmente el tratamiento indicado, mientras 34,4\% incumplieron el tratamiento en alguna medida $\left(\chi^{2}=6,250 ; \mathrm{P}=0,012\right)$. Según el análisis de regresión se encontró que solamente la combinación de niveles intermedios de estrés vinculado con la tolerancia a la ambigüedad y niveles bajos de depresión presentó una asociación significativa $(F[3,58]=3,298 ; \mathrm{P}=0,027)$ con la adhesión al tratamiento; la combinación de ambas variables explicó $38,2 \%$ de la varianza total encontrada.

Conclusiones. La combinación de los niveles de estrés vinculado con la tolerancia a la ambigüedad y de depresión podría emplearse como factor de predicción del fiel cumplimiento de las indicaciones médicas prescritas. Se deben tener en cuenta estos resultados al diseñar intervenciones y programas dirigidos a promover la adhesión al tratamiento en personas seropositivas al VIH.

Palabras clave Estrés, depresión, cooperación del paciente, VIH, México.

Investigador independiente, Hermosillo, Sonora, México. La correspondencia se debe dirigir a Julio A. Piña López, Calle Guillermo Prieto No. 18, Col. Constitución, Hermosillo, Sonora, C.P. 83150, México. Correo electrónico: ja_pina@hotmail.com

2 Facultad de Psicología, Universidad Nacional Autónoma de México, México, D.F., México.

3 Centro Ambulatorio para la Prevención y Atención a VIH/Sida e ITS, Secretaría de Salud Pública del Estado de Sonora, Sonora, México.
En la actualidad se han acumulado suficientes datos acerca de cómo los trastornos psicológicos interfieren con el desempeño de diversas actividades y algunos comportamientos, entre ellos los relacionados con la adhesión a los tratamientos prescritos por los médi- cos para controlar la infección por el virus de la inmunodeficiencia humana (VIH). Entre los trastornos que afectan de manera especial a la adhesión al tratamiento de las personas seropositivas al VIH - y por ende al curso clínico de la infección- destacan el es- 
trés percibido (1-3), la depresión (4-6) y la baja motivación para seguir el tratamiento prescrito $(7,8)$.

Sin embargo, las investigaciones relacionadas con la falta de adhesión al tratamiento en estas personas no se han basado en modelos teóricos articulados que permitan explicar cómo operan estos trastornos psicológicos y cuáles son los efectos que producen, tanto con respecto a la adhesión al tratamiento en sí como a la evolución del estado general de salud de las personas seropositivas. Una base teórica sólida y explícita suele ser un requisito indispensable para interpretar adecuadamente los resultados de las intervenciones interdisciplinarias dirigidas a promover la adhesión consistente y eficaz al tratamiento $(9,10)$.

De acuerdo con un modelo psicológico publicado recientemente (11), el estrés en las personas seropositivas al VIH se define como una reacción generalizada, cuyas reacciones específicas en el plano biológico - como la liberación de la hormona corticotrópica, la estimulación de las glándulas suprarrenales y la liberación de catecolaminas y glucocorticoides- y sus concurrentes psicológicas - como la sensación de tensión y ansiedad - dependen de la intensidad o la duración de los estímulos que esas personas enfrentan en diferentes situaciones (figura 1). Por lo tanto, el estrés es una variable de proceso que se inscribe en la primera fase de este modelo teórico y se caracteriza por: a) lo ambiguo de las señales y los eventos de estímulo a los que hay que responder —ambigüedad-; b) lo impredecible de las consecuencias relacionadas con las respuestas -impredecibilidad-; y c) la independencia de las consecuencias $-\mathrm{O}$ la ausencia de estas-con respecto al comportamiento de una persona en un momento dado del proceso de interacción -incertidumbre (12).

Los motivos, como variable, se refieren a la elección o la preferencia de determinados objetos, eventos o personas con los que se entra en contacto en situaciones socialmente valoradas (13, 14). Las personas con VIH pueden sentirse motivadas a seguir adecuadamente el tratamiento si en un momento dado consideran que es oportuno y pertinente hacerlo. En particular, esto suele ocurrir cuando las personas seropositivas asocian la adhesión al tratamiento con determinadas consecuencias positivas relacionadas con su propio estado de salud
- por ejemplo, cuando perciben una mejoría en su estado físico o psicológico- o cuando esto les genera elogios y muestras de afecto de personas importantes de su entorno social inmediato, entre otras consecuencias.

Por su parte, la depresión constituye una categoría de análisis general que forma parte de los comportamientos asociados con la condición de seropositivos de estas personas (tercera fase del modelo). La depresión se caracteriza por una amplia gama de comportamientos —el aislamiento social, la tristeza, la percepción de aceptación o rechazo por parte de otras personas importantes de su entorno social, la ira, la impulsividad, etcétera- que se derivan del diagnóstico de la infección o del tratamiento antirretroviral administrado.

De acuerdo con las hipótesis teóricas del modelo, se espera que en la medida en que una persona seropositiva al VIH experimente bajos niveles de estrés y depresión y se encuentre altamente motivada, aumente la probabilidad de que se desempeñe adecuadamente $y$, por ende, siga su tratamiento - en particular la toma de medicamentos- y abandone el consumo de alcohol y drogas. Si los niveles de es-

FIGURA 1. Modelo psicológico para la investigación del comportamiento de adhesión al tratamiento en personas seropositivas al VIH

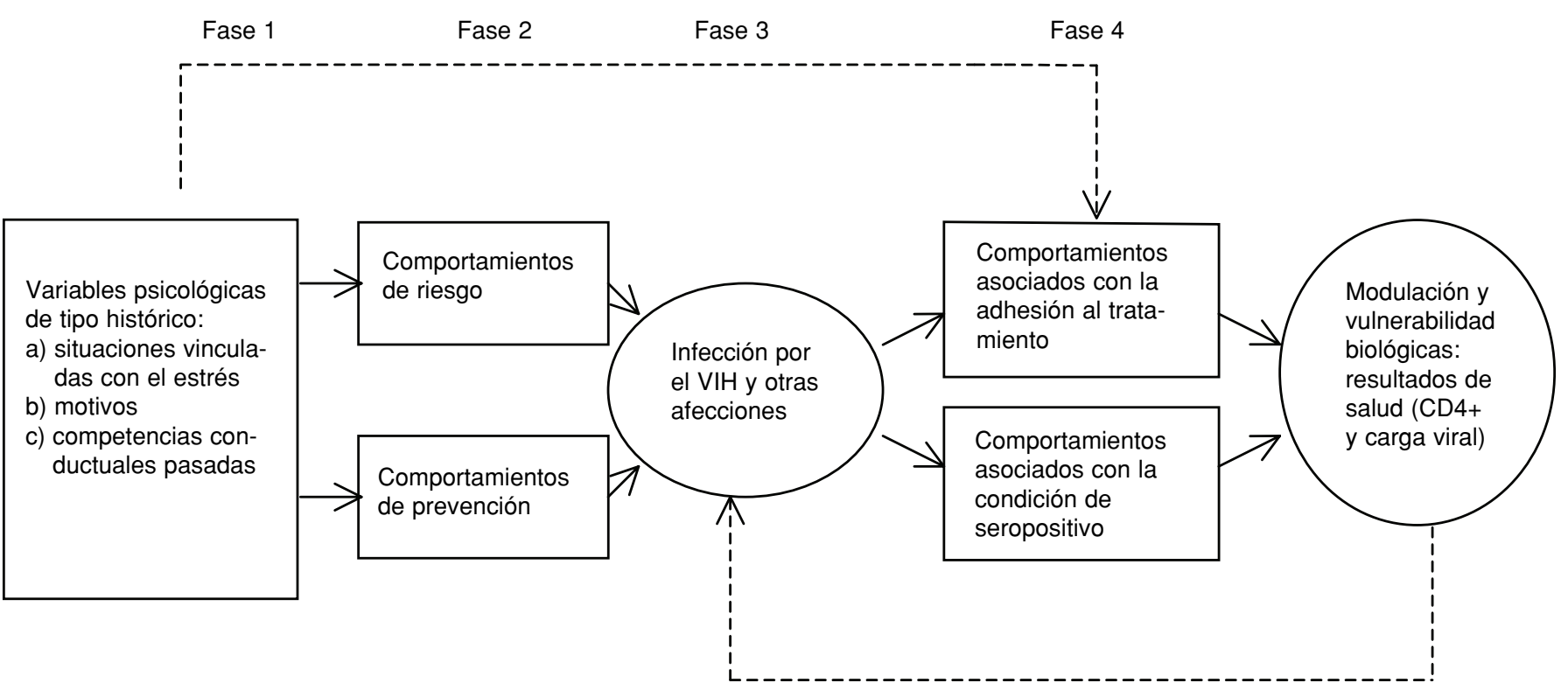


trés o de depresión son elevados o la persona no se siente motivada aumentará la probabilidad de que baje el desempeño de sus competencias $y$, por tanto, se alejará de la práctica consistente y eficaz del comportamiento de adhesión $(11,12)$.

El objetivo del presente estudio fue evaluar, desde la perspectiva de un modelo psicológico teórico, la asociación entre las variables relacionadas con el estrés, los motivos y la depresión en personas seropositivas al VIH y la adhesión al tratamiento, y analizar su consistencia según el modelo planteado.

\section{MATERIALES Y MÉTODOS}

Se realizó un estudio transversal en el Centro Ambulatorio para la Prevención y Atención a VIH/Sida e Infecciones de Transmisión Sexual (CAPASITS) de la ciudad de Hermosillo, capital del Estado de Sonora, en el noroeste de México. Para ello se invitó a participar en este estudio - vía telefónica o por correo certificado- a las 125 personas seropositivas al VIH tipo I que recibían atención especializada en el CAPASITS en abril de 2007. Los criterios de inclusión fueron: a) tener 18 años de edad o más; b) haber recibido un diagnóstico de seropositividad al VIH al menos seis meses antes de aplicada la encuesta; c) contar con los datos sobre el conteo de linfocitos $\mathrm{T}-\mathrm{CD} 4+\mathrm{y}$ los niveles de carga viral; $\mathrm{d}$ ) encontrarse bajo tratamiento con medicamentos antirretrovirales, y e) expresar su consentimiento informado por escrito.

La muestra quedó finalmente conformada por 64 personas $(51,2 \%$ del total de personas seropositivas al VIH invitadas); de ellas 39 (60,9\%) eran hombres y $25(39,1 \%)$ eran mujeres; la edad promedio fue de 36,9 años. Se excluyeron 61 personas: 27 porque no se encontraban en tratamiento con medicamentos antirretrovirales y 34 porque no cumplieron con alguno de los restantes criterios de inclusión. En el cuadro 1 se presentan las principales características sociodemográficas, clínicas y de comportamiento de la muestra de estudio.

\begin{tabular}{|c|c|c|}
\hline Variable & No. & $\%$ \\
\hline \multicolumn{3}{|l|}{ Sexo } \\
\hline Masculino & 39 & 60,9 \\
\hline Femenino & 25 & 39,1 \\
\hline \multicolumn{3}{|l|}{ Estado civil } \\
\hline Soltera/o & 31 & 48,4 \\
\hline Casada/o & 13 & 20,3 \\
\hline Unión libre & 7 & 10,9 \\
\hline Separada/o & 5 & 7,8 \\
\hline Viuda/o & 8 & 12,6 \\
\hline \multicolumn{3}{|l|}{ Escolaridad } \\
\hline No tienen estudios & 2 & 3,1 \\
\hline Primaria & 17 & 26,6 \\
\hline Secundaria & 24 & 37,5 \\
\hline Bachillerato & 12 & 18,7 \\
\hline Profesional & 9 & 14,1 \\
\hline \multicolumn{3}{|l|}{ Orientación sexual } \\
\hline Heterosexual & 37 & 57,8 \\
\hline Homosexual & 19 & 29,7 \\
\hline Bisexual & 3 & 4,7 \\
\hline No respondieron & 5 & 7,8 \\
\hline \multicolumn{3}{|l|}{ Linfocitos T-CD4+ } \\
\hline < 200 células/mL & 14 & 21,8 \\
\hline 201-400 células/mL & 17 & 26,6 \\
\hline > 400 células $/ \mathrm{mL}$ & 33 & 51,6 \\
\hline \multicolumn{3}{|l|}{ Carga viral } \\
\hline$<400$ copias $/ \mathrm{mL}$ & 22 & 34,4 \\
\hline 400-30 mil copias $/ \mathrm{mL}$ & 29 & 45,3 \\
\hline > 30 mil copias $/ \mathrm{mL}$ & 13 & 20,3 \\
\hline
\end{tabular}

Se utilizó el sistema de medición de variables psicológicas y comportamientos relacionados con la adhesión al tratamiento en personas seropositivas al VIH conocido por sus siglas VPAD-24 (15), que contiene preguntas específicas sobre la adhesión al tratamiento, como la cantidad y la frecuencia con que la persona toma los medicamentos $y$ las dificultades que experimenta para evitar el consumo de alcohol y drogas. Contiene también preguntas que exploran los motivos que influyen en estos comportamientos. La consistencia interna de estas preguntas fue alta ( $\alpha$ de Cronbach de 0,792 ).

Se utilizó la escala de situaciones vinculadas con el estrés SVE-12 (16) para explorar el estrés relacionado con la toma de decisiones (tres preguntas), la tolerancia a la ambigüedad (tres preguntas) y la tolerancia a la frustración (seis preguntas). El valor del coeficiente $\alpha$ de Cronbach para esta escala fue de 0,926 .

Por último, se utilizó la escala de depresión de Zung (EDZ-20), adaptada y validada para personas con VIH (17), que consta de 20 preguntas relacionadas con el ánimo depresivo y los síntomas físicos y psicológicos ( $\alpha$ de Cronbach de 0,887 ).

Se utilizó el paquete estadístico SPSS v. 15.0 para Windows para calcular las frecuencias y los porcentajes de las variables discretas y un índice que resumía las puntuaciones promedio de las respuestas relacionadas con las variables estrés, motivos y depresión. Se empleó la prueba de la ji al cuadrado de Pearson para establecer las 
posibles diferencias significativas entre los participantes que cumplieron cabalmente su tratamiento (adhesión total) y los que no lo cumplieron en alguna medida. Finalmente, el análisis de regresión múltiple (método de eliminación de variables hacia atrás) permitió evaluar la posible asociación entre las variables vinculadas con el estrés, los motivos y la depresión en esta muestra con la adhesión, mediante la eliminación de las variables cuyo valor de $F<2,71$. Para probar la bondad de ajuste de los datos al modelo lineal se empleó el coeficiente de determinación $R^{2}$ ajustado (18). Se escogió un nivel de significación $P \leq 0,05$.

Esta investigación recibió la aprobación del Comité de Investigación y Calidad de la Secretaría de Salud Pública del Estado de Sonora, México.

\section{RESULTADOS}

Los índices que resumen las puntuaciones promedio de cada una de las variables independientes - estrés, depresión y motivos- presentaron valores bajos o intermedios (cuadro 2). En cuanto a las variables de estrés, las puntuaciones reflejaron niveles intermedios (estrés vinculado con la toma de decisiones: 14,34; estrés vinculado con la tolerancia a la ambigüedad: 17,04; y estrés vinculado con la tolerancia a la frustración: 27,43). El índice de motivación $(14,01)$ se acercó al valor máximo esperado $(16,00)$, lo que cons- tituye un elemento positivo, ya que una mayor puntuación refleja una mayor motivación. Por último, el nivel de depresión observado fue bajo $(30,61)$.

Con respecto a la adhesión al tratamiento durante el mes anterior de aplicada la encuesta, $22(34,4 \%)$ de los 64 participantes no cumplieron las indicaciones en alguna medida y 42 $(65,6 \%)$ las siguieron en su totalidad $\left(\chi^{2}=6,250 ; P=0,012\right)$.

Al examinar mediante el análisis de regresión múltiple las tres variables independientes o sus combinaciones para probar su asociación con la adhesión al tratamiento (cuadro 3), se encontró que solamente la combinación de niveles intermedios de estrés vinculado con la tolerancia a la ambigüedad y bajos de depresión presentó una asociación significativa $(F[3,58]=3,298$; $P=0,027)$. La combinación de ambas variables explicó $38,2 \%$ de la varianza total encontrada $\left(R^{2}\right.$ ajustado $\left.=0,382\right)$.

\section{DISCUSIÓN}

Los resultados del presente estudio corroboran el papel benéfico que desempeñan los niveles bajos o intermedios de estrés y los bajos de depresión sobre la adhesión al tratamiento en personas seropositivas al VIH. Si se toma en cuenta que la adhesión al tratamiento constituye el eslabón final de una secuencia de interacciones -es decir, de comportamientos que se practican con respecto a uno mismo, a

CUADRO 2. Puntuaciones de los índices de las variables psicológicas en personas seropositivas al VIH, México, 2007

\begin{tabular}{|c|c|c|c|c|}
\hline \multirow[b]{2}{*}{ Índices } & \multirow{2}{*}{$\begin{array}{c}\text { Puntuación } \\
\text { obtenida }\end{array}$} & \multirow{2}{*}{$\begin{array}{l}\text { Desviación } \\
\text { estándar }\end{array}$} & \multicolumn{2}{|c|}{$\begin{array}{c}\text { Puntuaciones } \\
\text { mínimas y máximas }\end{array}$} \\
\hline & & & Observadas & Esperadas \\
\hline Motivos & 14,01 & 2,24 & $4-16$ & $4-16$ \\
\hline \multicolumn{5}{|l|}{ Estrés relacionado con la toma } \\
\hline de decisiones & 14,34 & 9,66 & $3-30$ & $3-30$ \\
\hline \multicolumn{5}{|l|}{ Estrés relacionado con la tolerancia } \\
\hline a la ambigüedad & 17,04 & 10,52 & $3-30$ & $3-30$ \\
\hline \multicolumn{5}{|l|}{ Estrés relacionado con la tolerancia } \\
\hline a la frustración & 27,43 & 16,47 & $3-60$ & $3-60$ \\
\hline Depresión & 30,61 & 9,57 & $20-68$ & $20-80$ \\
\hline
\end{tabular}

otras personas importantes del entorno social inmediato o al personal de salud a lo largo del proceso de interacción- (19), se puede comprender la importancia vital que tiene la identificación de las variables psicológicas que facilitan o dificultan esas interacciones o comportamientos. Esta información es de suma importancia para diseñar intervenciones interdisciplinarias específicas que conduzcan a un mejor ajuste psicológico y social de estas personas con relación a su condición de seropositivos y a la prolongación de su tiempo de vida con una mejor calidad $(10,20,21)$.

Las personas seropositivas al VIH que participaron en el presente estudio presentaron puntuaciones promedio intermedias en las preguntas relacionadas con el estrés y puntuaciones bajas en las relacionadas con la depresión, lo que indica que, en términos generales, este grupo de personas se ha ajustado favorablemente a su condición y a su tratamiento (22-24).

En el caso concreto del estrés vinculado con la tolerancia a la ambigüedad, los resultados indican que se trata de un grupo de personas que ha aprendido a reconocer que entre la demanda - es decir, consumir los medicamentos- y las consecuencias favorables asociadas con la adhesión - percepción de bienestar físico y psicológico, por ejemplo- puede pasar un tiempo considerable. Ese tiempo puede variar de una persona a otra y depende, entre otras cosas, del lapso transcurrido desde el diagnóstico de seropositividad al VIH, de los antecedentes clínicos - especialmente de algunas afecciones asociadas con esta infección-, del tipo de tratamiento empleado o de la cantidad de medicamentos que se consume diariamente (25-27).

En la medida en que las personas seropositivas al VIH reconocen la correspondencia que hay entre la demanda y las consecuencias positivas en el mediano o largo plazos, será menor la probabilidad de que se generen estados de estrés por lo ambiguo de esta situación. Esto último es particularmente importante si se toma en cuenta que las consecuencias positivas y sus efectos 
CUADRO 3. Resultados del análisis de regresión lineal múltiple de las variables psicológicas relacionadas con la adhesión al tratamiento en personas seropositivas al $\mathrm{VIH}$, México, 2007

\begin{tabular}{lccc}
\hline \multicolumn{1}{c}{ Variables } & $\beta$ & $t^{\text {a }}$ & $P^{\mathrm{a}}$ \\
\hline Estrés y tolerancia a la ambigüedad & 0,399 & 1,988 & 0,052 \\
Estrés y tolerancia a la frustración & $-0,349$ & $-1,74$ & 0,087 \\
Depresión & $-2,281$ & $-2,308$ & 0,025 \\
\hline
\end{tabular}

a Constante: $t=13,583 ; P<0,001$

en el plano biológico -incremento en los conteos de linfocitos T-CD4+ y reducción de los niveles de carga viralno siempre son inmediatos (28-30). Es justamente por ello que es fundamental que se provea de información oportuna a las personas seropositivas, como parte de las intervenciones interdisciplinarias, para que comprendan mejor qué es lo que se debe esperar y qué tiempo pueden demorar las señales positivas, una vez que inician el tratamiento antirretroviral.

En ese mismo sentido se debe reconocer el papel que desempeña la depresión. Diversos estudios indican que las personas seropositivas al VIH que experimentan estados de depresión más o menos prolongados tienen menos probabilidades de seguir fielmente el tratamiento $y$, con el paso del tiempo, experimentan más problemas de salud (31-32). El bajo nivel de depresión encontrado en los participantes en este estudio puede indicar que estos se han ido ajustando psicológica y socialmente, tanto a su condición de seropositivos al VIH como al tratamiento médico.

El tiempo promedio de infección transcurrido desde el diagnóstico de la infección por el VIH de los participantes en este estudio (60,7 meses) puede explicar, al menos en parte, las puntuaciones observadas en estrés y depresión. Un estudio anterior demostró que el tiempo de infección influye en la adhesión al tratamiento y en sus factores psicológicos de predicción (33). Debido a que las personas de la muestra estudiada llevaban más de 5 años con la infección por el VIH, se puede suponer que una gran parte de ellas había podido superar los problemas psicológicos que suelen experimentar estas personas después del diagnóstico, tanto en el ámbito personal como en el de las relaciones con otras personas importantes de su entorno social $(4,34-35)$.

Aunque no se dispone de datos categóricos al respecto, no se puede descartar la influencia de los programas de intervención que habitualmente se llevan a cabo en el CAPASITS previo al ingreso de las personas infectadas por el VIH a los programas específicos de adhesión al tratamiento. Estas intervenciones, en las que se trabaja tanto a nivel personal como en grupos, están dirigidas a promover un mejor ajuste psicológico y una mayor cohesión social mediante técnicas conductuales orientadas a modificar algunos comportamientos asociados con la infección por el VIH, como la depresión, la ansiedad, la impulsividad y el aislamiento social, entre otros.

Los resultados de este estudio corroboran la pertinencia empírica del modelo empleado y la capacidad de predicción favorable que tienen los niveles bajos o intermedios de estrés y bajos de depresión con respecto a la adhesión al tratamiento. Sin embargo, se deben tener en cuenta tres limitaciones. En primer lugar, más de la mitad de las personas de la muestra recibieron tratamiento antirretroviral en una sola institución (el CAPASITS). En segundo lugar, llama la atención la influencia prácticamente nula de las restantes variables independientes en particular de las relacionadas con los motivos y el estrés asociado con la toma de decisiones y la tolerancia a la frustración-, por lo que se encuentran en proceso de revisión. ${ }^{4}$ Por úl- timo, es posible que los niveles intermedios de estrés y los bajos de depresión se deban a la influencia de otras variables no consideradas en este estudio, como el tiempo de infección, el tratamiento psicológico aplicado a las personas seropositivas al VIH y la influencia de otras personas del entorno social, como las parejas, los familiares o los amigos cercanos y el apoyo percibido (36-37).

No obstante esas limitaciones, el estudio brinda información valiosa, especialmente en dos sentidos. Primero, se ha probado un modelo para evaluar tempranamente el papel de dos condiciones psicológicas: el estrés -mediante el índice de estrés como variable de proceso-, que aparece en la primera fase y es clave antes y después del diagnóstico de la infección por el VIH, y la depresión -mediante el índice de depresión como variable de resultado-, que suele aparecer después del diagnóstico. Estas condiciones afectan negativamente el ajuste de las personas tanto en el plano personal como social. Segundo, se ha confirmado que las personas seropositivas al VIH pueden responder de manera adecuada y oportuna a los requerimientos que imponen tanto la infección como el propio tratamiento.

De acuerdo con los resultados del presente estudio, la muestra analizada presentó una adecuada adhesión al tratamiento antirretroviral. Se encontró que la combinación de niveles intermedios de estrés vinculado con la tolerancia a la ambigüedad y bajos de depresión presentó una asociación significativa con la adhesión al tratamiento y podría emplearse como factor de predicción del fiel cumplimiento de las indicaciones médicas prescritas.

Estos resultados se deben tener en cuenta al diseñar intervenciones y pro-

\footnotetext{
4 Piña JA, Sánchez-Sosa JJ, Corrales AE, Togawa C. Variables psicológicas y comportamientos de adhesión en personas con VIH: estudio prospectivo y longitudinal a los seis meses. Salud Publica Mex (en revisión); Sánchez-Sosa JJ, Piña JA, Corrales AE, Valencia MA, Vázquez RL. Aplicación de un modelo psicológico a la investigación de los comportamientos de adhesión terapéutica en personas con VIH. Salud Mental (en revisión).
} 
gramas dirigidos a promover la adhesión al tratamiento en personas seropositivas al VIH. Se recomienda emprender estudios similares con una muestra mayor de personas atendidas en otras instituciones de salud, así como añadir otras variables que puedan influir en el grado de adhesión de estas personas al tratamiento, en particular el apoyo social percibido. Asi- mismo, se debe ampliar el análisis de las variables de comportamiento y de los posibles factores psicológicos de predicción incorporados al modelo, ajustados por el tiempo de infección.

\section{REFERENCIAS}

1. Remor E, Penedo FJ, Shen BJ, Schneiderman $\mathrm{N}$. Perceived stress is associated with CD4+ cell decline in men and women living with HIV/AIDS in Spain. AIDS Care. 2007;19: 215-9.

2. Weaver KE, Llabre MM, Durán RE, Antoni $\mathrm{MH}$, Ironson G, Penedo FJ, et al. A stress and coping model of medication adherence and viral load in HIV-positive men and women on highly active antiretroviral therapy (HAART). Health Psychol. 2005;24:385-92.

3. Siegel K, Schrimshaw EW. The stress moderating role of benefit finding on psychological distress and well-being among women with HIV/AIDS. AIDS Behav. 2007;11:421-33.

4. Cook JA, Grey D, Burke-Miller J, Cohen MH, Anastos K, Ghandi M, et al. Effects of treated and untreated depressive symptoms on highly active antiretroviral therapy use in a US multi-site cohort of HIV-positive women. AIDS Care. 2006;18:93-100.

5. Teva I, Bermúdez MP, Hernández-Quero J, Buela-Casal G. Evaluación de la depresión, ansiedad e ira en pacientes con VIH/SIDA. Salud Mental. 2005;28:40-9.

6. Ironson $\mathrm{G}, \mathrm{O}^{\prime}$ Cleirigh $\mathrm{C}$, Fletcher MA, Laurenceau JP, Balbín E, Klimas N, et al. Psychosocial factors predict CD4 and viral load change in men and women with human immunodeficiency virus in the era of highly active antiretroviral therapy. Psychosom Med. 2005;67:1013-21.

7. Södegard B, Halvarsson M, Lindbäck S, Sönneborg A, Tully MP, Lindblad AK. Differences in adherence and motivation to HIV therapy-two independent assessments in 1998 and 2002. Phar World Ther. 2006;28:248-56.

8. García R, Pondé M, Lima M, de Souza AR, de Stolze MO, Badaró R. Lack of motivation on the adherence of HIV-positive/AIDS patients to antiretroviral therapy. Braz J Inf Dis. 2005;9:494-9.

9. Hotz S, Kaptein A, Pruitt S, Sánchez-Sosa JJ, Wiley C. Behavioural mechanism explaining adherence. What every health professional should know. In: WHO, ed. Adherence to long-term therapies. Evidence for action. Geneva: WHO; 2003. Pp. 157-71.

10. Sánchez-Sosa JJ. Treatment adherence: the role of behavioral mechanism and some implications for health care interventions. Rev Mex Psicol. 2002;19:85-92.

11. Piña JA, Sánchez-Sosa JJ. Modelo psicológico para la investigación de los comportamientos de adhesión en personas con VIH. Universitas Psychol. 2007;3:399-407.

12. Ribes E. Psicología y salud: un análisis conceptual. Barcelona: Martínez Roca; 1990.
13. Ribes E. ¿Qué es lo que se debe medir en psicología?: la cuestión de las diferencias individuales. Acta Comportam. 2005;13:37-52.

14. Ribes E, Sánchez S. El problema de las diferencias individuales: un análisis conceptual de la personalidad. En: Ribes E, ed. Problemas conceptuales en el análisis del comportamiento. México, D.F.: Trillas; 1990. Pp. 79-99.

15. Piña JA, Corrales AE, Mungaray $K$, Valencia MA. Instrumento para medir variables psicológicas y comportamientos de adhesión al tratamiento en personas seropositivas frente al VIH (VPAD-24). Rev Panam Salud Publica. 2006;19:217-28

16. Piña JA, Valencia MA, Mungaray $K$, Corrales AE. Validación de una escala breve que mide situaciones vinculadas con estrés en personas VIH positivas. Terapia Psicol. 2006;24:15-21.

17. Rivera BM, Piña JA, Cázarez O, Corrales AE. Validación de la escala de depresión de Zung en personas con VIH. Terapia Psicol. 2007;25:135-40.

18. Silva A. La investigación asistida por computadora. México, D.F.: Universidad Nacional Autónoma de México; 1998.

19. Bayés R. Psicología de la salud: dos preguntas en busca de respuesta. Anuario Psicol. 1998;29:167-9.

20. Ammassari A, Antinori A, Aloisi MS, Trotta MP, Murri R, Bartoli L, et al. Depressive symptoms, neurocognitive impairment, and adherence to highly active antiretroviral therapy among HIV-infected persons. Psychosom. 2004;45:399-402.

21. Amico KR, Harman JJ, Johnson BT. Efficacy of antiretroviral therapy adherence interventions: a research synthesis of trials, 1996-2004. J Acquir Immune Defic Syndr. 2006;41:285-97.

22. Malcolm SE, Ng JJ, Rosen RK, Stone VE. An examination of HIV/AIDS patients who have excellent adherence to HAART. AIDS Care. 2003;15:251-61.

23. Heckman BD, Catz SL, Heckman TG, Miller JG, Kalichman SC. Adherence to antiretroviral therapy in rural persons living with HIV disease in the United States. AIDS Care. 2004;16:219-30.

24. Van Dulmen S, Sluijs E, van Dijk L, de Ridder $\mathrm{D}$, Heerdink R, Bensing J. Patient adherence to medical treatment: a review of reviews. BMC Health Services Res. 2007;7(55).

25. Olley BO, Zeier MD, Seedat S, Stein DJ. Posttraumatic stress disorder among recently diagnosed patients with HIV/AIDS in South Africa. AIDS Care. 2005;17:550-7.

26. Kleeberger CA, Phair JP, Stathdee SA, Detels R, Kingsley L, Jacobson LP. Determinants of heterogeneous adherence to HIV-antiretrovi- ral therapies in the multicenter AIDS cohort study. J AIDS. 2001;26:82-92.

27. Park WB, Choe PG, Kim SH, Ho JH, Bang JH, Kim HB, et al. One-year adherence to clinic visits after highly active antiretroviral therapy: a predictor of clinical progress in HIV patients. J Int Med. 2007;261:268-75.

28. Moore AL, Mocroft A, Madge S, Devereaux H, Wilson D, Phillips AN, et al. Gender differences in virologic response to treatment in an HIV-positive population: a cohort study. JAIDS. 2001;26:159-63.

29. Breton G, Lewden C, Spire B, Salmon D, BrunVézinet $\mathrm{F}$, Duoung $\mathrm{M}$, et al. Characteristics and response to antiretroviral therapy of HIV-1-infected patients born in Africa and living in France. HIV Med. 2007;8:164-70.

30. Thiébaut $\mathrm{R}$, Jacqmin-Gadda $\mathrm{H}$, Walker $\mathrm{S}$, Sabin C, Prins M, del Amo J, et al. Determinants of response to first HAART regimen in antiretroviral-naïve patients with an estimated time since HIV seroconversion. HIV Med. 2006;7:1-9.

31. Grierson JW, Pitts MK, Misson S. Health and well-being of HIV-positive Australians: findings from the third national HIV feature survey. Int J STD AIDS. 2005;16:802-6.

32. Nogueira L, de Fátima P, Crosland MD. Anxiety and depression assessment prior to initiating antiretroviral treatment in Brazil. AIDS Care. 2006;18:529-36.

33. Piña JA, Rivera BM, Corrales AE, Mungaray $\mathrm{K}$, Valencia MA. ¿Influye el tiempo de infección en meses sobre predictores psicológicos de comportamientos de adhesión en personas con VIH? Terapia Psicol. 2006;24:183-90.

34. Godin G, Coté C, Naccache H, Lambert D, Trottier S. Prediction of adherence to antiretroviral therapy: a one-year longitudinal study. AIDS Care. 2005;17:493-504.

35. Sun HM, Zhag JJ, Fu XP. Psychological status, coping, and social support of people living with HIV/AIDS in central China. Public Health Nurs. 2007;24:132-40.

36. Johnson MO, Elliot TR, Neilands TB, Morin SF, Chesney MA. A social problem-solving model of adherence to HIV medication. Health Psychol. 2006;25:355-63.

37. Simoni JM, Frick PA, Huang B. A longitudinal evaluation of a social support model of medication adherence amonh HIV-positive men and women on antiretroviral therapy. Health Psychol. 2006;25:74-81.

Manuscrito recibido el 18 de mayo de 2007. Aceptado para publicación, tras revisión, el 5 de marzo de 2008. 
ABSTRACT Objectives. To evaluate the association between variables related to stress, reasons, and depression, and adherence to treatment in HIV-positive individuals, and to analyze the robustness according to a theoretical psychology model.

\section{Association between stress and depression levels and treatment adherence among HIV-positive individuals in Hermosillo, México}

Methods. This was a cross-sectional study of 25 female and 39 male HIV-positive participants receiving services at the Outpatient Center for Prevention and Treatment of HIV / AIDS and Sexually-transmitted Diseases (Centro Ambulatorio para la Prevención y Atención del VIH/Sida e Infecciones de Transmisión Sexual) in Hermosillo, Sonora, Mexico. The psychological variables, the degree of treatment adherence, stress-related circumstances, and the severity of depression were examined. Rates were calculated for the key variables associated with stress, reasons, and depression. The associations among variables were determined by multiple regression.

Results. During the month preceding the study, $65.6 \%$ of the 64 participants reported having faithfully followed the prescribed treatment, while $34.4 \%$ had failed, in some way, to follow the treatment $\left({ }^{-} 2=6.250 ; \mathrm{P}=0.012\right)$. Regression analysis found that there was only one combination, an intermediate level of stress linked to tolerance of uncertainty and low levels of depression, that was significantly associated ( $\mathrm{F}$ $[3.58]=3.298 ; \mathrm{P}=0.027$ ) with adherence to treatment; the combination of these two variables explained $38.2 \%$ of the total variance found.

Conclusions. The combination of stress levels with tolerance of uncertainty and depression could be used as a predictor for true compliance with prescribed treatment plans. These results should be taken into account when designing intervention and treatment-adherence campaigns in HIV-positive individuals.

Key words Stress, depression, patient compliance, HIV, Mexico.

\begin{tabular}{|c|c|}
\hline \multicolumn{2}{|c|}{ Atención comunitaria a personas con trastornos psicóticos } \\
\hline $\begin{array}{c}\text { Atención comunitaria } \\
\text { a personas con } \\
\text { trastornos psicóticos }\end{array}$ & $\begin{array}{l}\text { En este libro se presentan } 13 \text { guías operativas para desarrollar programas comuni- } \\
\text { tarios de atención a personas con trastornos psicóticos que se puedan integrar a } \\
\text { los planes nacionales de salud mental. La primera parte de esta obra presenta las } \\
\text { bases epidemiológicas y científicas de los trastornos mentales, así como las estrate- } \\
\text { gias utilizadas en la formulación de programas de atención comunitaria para } \\
\text { quienes los padecen. La segunda parte la constituyen las guías operativas, en las } \\
\text { que se recomiendan medidas para desarrollar mejores programas de atención } \\
\text { comunitaria en el marco de la salud mental. }\end{array}$ \\
\hline 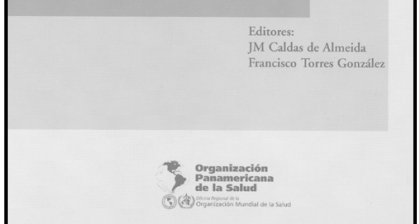 & $\begin{array}{l}\text { Esta publicación será de gran utilidad para los responsables de formular políticas } \\
\text { de salud mental, médicos encargados de las unidades de salud mental en las } \\
\text { diversas instituciones de atención comunitaria de salud, psiquiatras, psicólogos, } \\
\text { personal de enfermería, proveedores de atención comunitaria, estudiantes de psi- } \\
\text { cología, psiquiatría y salud pública, así como para lectores interesados en la salud }\end{array}$ \\
\hline $\begin{array}{l}\text { 2005,152 pp. } \\
\text { ISBN } 9275316015 \\
\text { Código: PC } 601 \\
\text { Precio: US } \$ 18.00 \text { en América } \\
\text { Latina y el Caribe/ US } \$ 25.00 \text { en } \\
\text { el resto del mundo }\end{array}$ & $\begin{array}{l}\text { Adquiera esta publicación por medio de la librería en línea de la OPS: } \\
\text { http://publications.paho.org; correo electrónico: paho@pmds.com; } \\
\text { Fax: (301) 209-9789; Oficina de país de la OPS/OMS }\end{array}$ \\
\hline
\end{tabular}

